\title{
Coloni et incolae, vingt ans après ${ }^{1}$ Mobilité et identité sociales et juridiques dans le monde romain occidental
}

\author{
Stéphane Benoist
}

Il m'apparaît nécessaire de rappeler que toute histoire, consciemment ou inconsciemment, s'écrit au temps présent et qu'il est ainsi illusoire de considérer que le contexte de rédaction puisse n'avoir aucune incidence sur la nature des enquêtes historiques que nous menons. ${ }^{2}$ Dans les années 1970-1980, l'essentiel de la réflexion a porté, tant en France que dans le monde anglo-saxon, sur la définition des empires et de l'impérialisme, ce qui induisait un rapport étroit entre histoire contemporaine de la décolonisation et perception du monde romain impérial, l'une et l'autre questions étant étroitement liées en Afrique du Nord romaine. ${ }^{3}$ Il ne viendrait à personne de considérer qu'un long

1 Je tiens à remercier les éditeurs du volume pour leurs fructueuses remarques. Le titre de ce chapitre salue les uicennalia de la parution de l'article d'A. Chastagnol, 'Coloni et incolae. Notes sur les différenciations sociales à l'intérieur des colonies romaines de peuplement dans les provinces de l'Occident ( ${ }^{\text {er }}$ s. av. J.-C.- ${ }^{\text {er }}$ s. ap. J.-C.)', in A. Chastagnol, S. Demougin et C. Lepelley, eds., Splendidissima civitas. Études en hommage à François Jacques (Paris 1996), 13-25 = idem, La Gaule romaine et le droit latin. Recherches sur l'histoire administrative et sur la romanisation de ses habitants. Scripta varia 3. Coll. du Centre d'études romaines et galloromaines 4 (Lyon 1995), 131-141.

2 Une seule référence suffira: les multiples entrées du récent Dictionnaire de l'historien (Paris 2015), notamment celles abordant la question du métier de l'historien et de ses pratiques, 'Historien' (C. Gauvard et J.-Fr. Sirinelli, 371-375), 'Temps', 'Antiquité', 'Périodisation' et 'Durée' (S. Benoist, 694-698, 22-23, 527-529 et 187-188). Et plus directement pour notre présent propos: 'Migrations' (M. Martini, 457-459) et 'Identité(s)' (C. Judde de Larivière, 384-386).

3 À propos des débats concernant l'impérialisme à Rome, partir en premier lieu de P. Veyne, 'Ya-t-il eu un impérialisme romain?', Mélanges de l'École française de Rome. Antiquité 87 (1975), 793-855, puis de deux colloques contemporains, français et américain: L'impérialisme romain. Histoire, idéologie, historiographie, publié en deux livraisons de la revue Ktèma 7 (1982), 141233 et 8 (1983), 111-277; notamment les contributions d'Ed. Frézouls, 'Sur l'historiographie de l'impérialisme romain', 141-162 et C. Nicolet, 'L'Empire romain: espace, temps et politique', 163-173; cf. auparavant la conclusion de ce dernier dans C. Nicolet ed., Rome et la conquête 
quatrième siècle qui nous mène de la mise en place de la tétrarchie au sac de Rome par Alaric, en nous livrant des récits historiques - les épitomés d'Eutrope, d'Aurelius Victor ou du Pseudo-Aurelius Victor -, des biographies impériales avec le de uita principum de l'Histoire Auguste ${ }^{4}$ des récits annalistiques comme l'Histoire romaine d'Ammien Marcellin, mais également des discours épidictiques, notamment les Panégyriques latins, et finalement les prémices d'une histoire universelle chrétienne avec Eusèbe ou Jérôme, n'est pas ancré dans un présent qui offre à l'historien matière à réfléchir sur la longue durée et son usage raisonné, du Principat à l'empire chrétien, par une nouvelle conception de la cité universelle et un nouvel équilibre, de part et d'autre des frontières d'un empire de plus en plus régulièrement franchies en tout sens... ${ }^{5}$ De la sorte, l'année 2015 aura fourni à tout Européen convaincu matière à de multiples interrogations quant aux conceptions politiques et territoriales aux contours fluctuants, ouvertes ou fermées, face à cette image poignante de migrants incarnant une nouvelle quête, l'Europe étant elle-même perpétuellement en recherche d'unité politique, sinon d'identité sociale. L'historien d'un Imperium Romanum le conduisant de la République médiane à l'Antiquité tardive ne s'égare nullement en ayant en tête cette brûlante actualité tout en réfléchissant à nouveaux frais sur la mobilité et les migrations dans le monde romain. Les propos qui suivent sont donc hic et nunc marqués par les temps présents et réaffirment la nécessité d'une mise en contexte permanente de tout écrit historique. Gageons qu'il n'est pas inutile de regarder le monde romain impérial depuis les rives d'une démocratie européenne désemparée, sinon aux abois!

du monde méditerranéen, t. II, Genèse d'un empire (Paris 1991), '1'impérialisme" romain', 883920; W.V. Harris, ed., The Imperialism of Mid-Republican Rome. Papers and Monographs of the American Academy in Rome 29 (Rome 1984), en particulier l'étude de J. Linderski, 'Si uis pacem, para bellum: Concepts of defensive imperialism', 133-164. En terminant par l'essai de J. Richardson, The Language of Empire. Rome and the Idea of Empire from the Third Century $B C$ to the Second Century $A D$ (Cambridge 2008). Pour un aperçu de l'historiographie française de l'Afrique du Nord, M. Dondin-Payre, 'Empire antique, empire contemporain: l'Afrique du Nord', in S. Benoist, A. Daguet-Gagey et Chr. Hoët-van Cauwenberghe, eds., Figures d'empire, fragments de mémoire. Pouvoirs et identités dans le monde romain impérial (II siècle avant notre ère-VI ${ }^{e}$ siècle de notre ère). Coll. Archaiologia (Villeneuve d'Ascq 2011), 49-70.

4 Pour reprendre la proposition de M. Thomson, 'The original title of the Historia Augusta', Historia 56 (2007), 123-125.

5 Quelques réflexions à paraître dans $\mathrm{S}$. Benoist, 'Biography, history and memory, about some imperial figures', All Souls College, 15 July 2015, Fergus Millar 8oth Birthday Celebration (London BICs Suppl.). 
Cette étude se propose de conduire une réflexion tout à la fois historiographique, méthodologique et prospective, en se fondant sur une documentation abordant prioritairement les statuts des hommes et des communautés sur un temps long, des héritages de la pratique républicaine - qui repose essentiellement sur les rapports entre Rome et les Latins, puis l'ensemble de la péninsule italienne, du cinquième au premier siècle avant notre ère - aux deux premiers siècles de l'empire, la constitutio Antoniniana représentant à bien des égards une étape, sinon un simple achèvement, d'un processus qui demeure une référence sans aucun équivalent dans la longue histoire des empires. ${ }^{6}$ La première partie de cette enquête porte sur une approche philologique et juridique de la mobilité à Rome et dans le monde romain, aux époques tardo-républicaine et impériale, en partant des termes polysémiques coloni et incolae. Le dossier des emplois épigraphiques de ces deux expressions, qui permet d'inverser les définitions naturelles des courants de la mobilité, du résident au migrant, conduit à aborder tout particulièrement dans un deuxième temps le problème de l'application du droit latin dans les communautés d'Occident. Une troisième partie conclusive revient sur les multiples conceptions de l'Imperium Romanum dans les réflexions récentes des historiens romanistes à propos de l'intégration, ou de l'homogénéité et de l'hétérogénéité de la société romaine impériale.

Le point de départ de cette enquête a été très clairement nourri par des travaux menés en France, dans les années 1980 et au tout début des années 1990. ${ }^{7}$ Des historiens et épigraphistes s'intéressaient aux statuts des hommes

6 C'est un élément fondamental insuffisamment commenté dans l'ouvrage récent tant débattu de J. Burbank et Fr. Cooper, Empires. De la Chine ancienne à nos jours (Paris 2011; trad. franç. de Empires in World History: Power and the Politics of Difference, Princeton, N.J. etc. 2010): voir de manière générale mes remarques dans $\mathrm{S}$. Benoist, 'Lecture et perspectives romaines', Monde(s), histoire, espaces, relations 2 (2012), 226-229, au sein de la rubrique 'Débat autour d'un livre'.

7 Mes premiers pas dans la recherche en France au milieu des années 1980 coïncident avec la tenue de séminaires, à l'École pratique des Hautes Études, section des Sciences historiques et philologiques, qui abordèrent des points essentiels pour mon propos et ont inspiré la reprise présente de ce travail. Il s'agit d'une part de la conférence de C. Nicolet qui exposait, à cette époque, les dossiers préparatoires à son maître livre sur L'inventaire du monde. Géographie et politique aux origines de l'Empire romain (Paris 1988 [2 éd. 1996]). On lira la présentation de cet ouvrage par M. Coudry, Cahiers du Centre Gustave Glotz 22 (2011), 111-122. D'autre part, le séminaire d'A. Chastagnol qui exploitait, avec la rigueur qui le caractérisait, la documentation épigraphique pour des études finalement regroupées dans son troisième volume de Scripta varia: La Gaule romaine et le droit latin, op. cit. (n. 1), paru il y a très exactement vingt ans. Si le premier abordait bien directement le problème de la mobilité, le second avait tout à fait à l'esprit l'importance de la dynamique constitutive de l'Imperium Romanum, en traitant des statuts des hommes et des communautés. 
et des communautés, mais également à la dynamique intégrative de l'empire romain et aux modes de fonctionnement politique et administratif de cette entité à nulle autre pareille, en ciblant la mémoire perdue des services romains ou les identités personnelles et collectives des cités de type romain, dont l'évolution dynamique s'apparente à une forme de cursus qui conduit de la cité pérégrine à la colonie romaine doté du ius Italicum. Cette capacité intégrative a dès lors inspiré les savants de toutes origines au cours des années 1990 et 2000, l'ensemble de la recherche tentant par des voies complémentaires et détournées de comprendre les spécificités du modèle romain. ${ }^{8}$ C'est donc bien dans une perspective ouverte sur la mobilité et les identités sociales et juridiques que je me place pour aborder dans ce qui suit un monde volontairement restreint à l'Occident romain, mais afin d'éclairer la compréhension d'un modèle d'empire hic et nunc.

2

\section{De la mobilité et des statuts: premières définitions. Peut-on parler d'identités' sociales et juridiques?}

Partons de cette proximité que je propose de retenir, en abordant tout à la fois la mobilité et les statuts, et rappelons brièvement quelques définitions qui sont autant de points de départ pour toute réflexion sur les manières romaines de penser la société civique, de définir la ciuitas. ${ }^{9}$ La circulation des hommes est apparue comme un enjeu qui cristallise les ressentiments de la part des alliés à l'égard des Romains et de leur évolution sensible entre les cinquième et quatrième siècles et la période postérieure, jusqu'à l'acmé d'une violente crise aux lendemains de la deuxième guerre punique qui finit par déboucher sur la guerre des socii et les dispositions juridiques qui s'en suivirent avec la

8 Un seul exemple suffira, avec la thèse de C. Ando, soutenue en 1996 et publiée en 200o: Imperial Ideology and Provincial Loyalty in the Roman Empire (Berkeley 2000).

9 C'est le premier objet d'étude de deux enquêtes récentes de C. Ando: L'Empire et le droit. Invention juridique et réalités historiques à Rome (Paris 2013), chap. 1 'Citoyens et étrangers devant la loi', et Roman Social Imaginaries. Language and Thought in Contexts of Empire (Toronto 2015), chap. 1 'Belonging'. On peut relever deux prémices à cette réflexion développées dans 'Law and the landscape of Empire', in S. Benoist, A. Daguet-Gagey et Chr. Hoëtvan Cauwenberghe, eds., Figures d'empire, fragments de mémoire. Pouvoirs et identités dans le monde romain impérial (II siècle avant notre ère-VI siècle de notre ère). Coll. Archaiologia (Villeneuve d'Ascq 2011), 25-47, et 'The Roman city in the Roman period', in S. Benoist, ed., Rome, a City and Its Empire in Perspective. The Impact of the Roman World through Fergus Millar's Research - Rome, une cité impériale en jeu. L'impact du monde romain selon Fergus Millar. Coll. Impact of Empire 16 (Leyde et Boston 2012), 109-124. 
municipalisation de l'Italie. ${ }^{10}$ Tout ceci est bien connu et ne sert que de toile de fond à ma réflexion. Je retiens donc, à partir d'une observation de Claude Nicolet qui s'intéressait tout particulièrement au contrôle de l'espace humain au moyen des recensements, que cette circulation des hommes domine largement le monde romain de l'époque républicaine avant que la circulation des documents signe une évolution majeure de l'organisation de l'Imperium Romanum et de la perception qu'il eut ou souhaita donner de lui-même. Je renvoie à ses analyses concernant cette connaissance du monde et aux deux citations suivantes pour appréhender la mobilité des hommes et le rôle du recensement: la remarque de Cicéron à propos de "cette foule venue de toute l'Italie pour les comices, les jeux, le recensement" et celle de Claude "combien une telle entreprise est pénible, nous le voyons bien maintenant par expérience, bien qu'il ne s'agisse aujourd'hui de rien d'autre que de faire connaître publiquement nos ressources." ${ }^{11}$ Il me semble que, dans une Italie devenue 'romaine' (nous sommes en 70), la remarque de Cicéron s'avère riche d'une lecture 'officielle' de ce qui fait le citoyen: comices, jeux et cens, à un moment où les hommes circulent encore, avant une décentralisation des opérations du cens dont nous informe la table d'Héraclée, les documents voyageant désormais pour des dénombrements statistiques centralisés à Rome, en lieu et place des hommes.

Il m’apparait, au regard de l'évolution de l'Italie et de sa lente mais irrésistible 'romanisation' au moyen du processus de municipalisation, que l'enjeu d'une mobilité sociale et juridique, qui s'était longtemps énoncée en termes de ius migrandi ou d'une acquisition per migrationem et censum, donc d'un déplacement effectif - physique - pour obtenir une promotion juridique, ${ }^{12}$ autre

10 L'une des meilleures approches et mises en perspective en français de ces phénomènes demeure les deux synthèses de J.-M. David, La Romanisation de l'Italie (Paris 1994) et La République romaine de la deuxième guerre punique à la bataille d'Actium, 218-31 av. J.-C. (Paris 2000).

11 Cic. I Verr. 54: Haec frequentia totius Italiae quae conuenit uno tempore undique comitiorum, ludorum, censendique causa; et la table claudienne de Lyon (CIL 13.1668 = ILS 212, II, 38-40): quod opus quam ar/duum sit nobis, nunc cum maxime, quamuis nihil ultra, quam/ ut publice notae sint facultates nostrae, exquiratur, nimis/ magno experimento cognoscimus; apud Nicolet 1988, op. cit. (n. 7), chapitre 6, 'Contrôle de l'espace humain: les recensements', 203, n. 18 et 199-200, 211, n. 66.

12 Se reporter à la mise au point récente (avec l'état bibliographique de la question) de D. Kremer, Ius Latinum. Le concept de droit latin sous la République et l'Empire (Paris 2006), 30-40, et en tout dernier lieu les réflexions de P. Le Roux, 'Le droit latin (ius latii): une relecture', in G. Cresci Marrone, ed., Trans Padum... usque ad Alpes. Roma tra il Po e le Alpi: dalla romanizzazione alla romanità (Rome 2015), 179-195, relecture de son désormais 
forme de déplacement, s'est transformé au profit d'une conception plus globale des différents statuts, de leur hiérarchie et d'un mouvement vers l'uniformisation. C'est en ce sens qu'il convient d'être attentif aux usages sémantiques, à la polysémie de certains termes et à l'ambiguïté de nos données concernant coloni et incolae, de la République à l'empire, tel que Yan Thomas tentait de les synthétiser en un volume très dense, dont je ne partage pas toutes les conséquences, notamment la conception évolutive du domicilium..$^{13}$ Assurément, les données dont nous disposons - littéraires, finalement assez réduites, épigraphiques, plus nombreuses mais parfois délicates à interpréter, et juridiques établissent une dualité structurelle (citoyens et résidents) qui rend compte d'une conception - certes à première vue traditionnelle de la cité antique, sinon constitutive, mais finalement plus ambiguë de la ciuitas, perçue, envisagée par les Romains. Des citoyens de plein droit (ciues, coloni ou municipes) cohabitent avec des résidents 'étrangers', au sens latin du terme peregrini, non-citoyens, mais dont l'origine a varié avec le temps, des ueteres incolae, les anciens habitants, les 'indigènes', aux incolae étrangers en résidence prolongée, ${ }^{14}$ qui n'ont pas cessé d'appartenir à leur ciuitas originelle.

Cette dualité des incolae se retrouve parfaitement dans toutes les études ayant abordé cette catégorie, des réflexions philologiques aux commentaires historiques, d'une définition du sens d'incola ${ }^{15}$ aux propos synthétiques d'une

classique article: 'Rome et le droit Latin', Revue historique de droit français et étranger 76.3 (1998), 315-341 = P. Le Roux, Espagnes romaines. L'empire dans ses provinces. Scripta Varia II, travaux rassemblés par S. Armani, F. Cadiou, et al. (Rennes 2014), 455-478.

13 Y. Thomas, 'Origine' et 'commune patrie'. Étude de droit public romain (89 av. J.-C.-212 ap. J.-C.). Coll. de l'École française de Rome 221 (Rome 1996). À ce propos, on renverra à la contribution de Claudia Moatti dans ce volume.

14 Ce critère de durée est formalisé sous Hadrien par la fixation d'une période de 10 ans: CJ 10. 40 (De incolis et ubi quis domicilium habere uidetur et de his qui studiorum causa in alia ciuitate degunt, "A propos des incolae, et pour tout lieu où un individu est censé avoir son domicilium, et à propos de ceux qui résident dans une autre cité en raison de la poursuite de leurs études"), 2 pr. (Imperator Alexander Severus. Nec ipsi, qui studiorum causa aliquo loci morantur, domicilium ibi habere creduntur, nisi decem annis transactis eo loci sedes sibi constituerunt, secundum epistulam diui Hadriani, nec pater, qui propter filium studentem frequentius ad eum commeat, "L'empereur Alexandre Sévère. Ceux qui demeurent dans quelque lieu que ce soit afin de poursuivre leurs études ne sont considérés comme ayant leur domicilium en ce lieu que s'ils y restent au moins dix ans. Selon une epistula du divin Hadrien, il n'en va pas de même pour un père qui visite fréquemment son fils, quand ce dernier poursuit ses études").

15 Définition du vocabulaire, à propos des coloni et incolae: le colonus est un "habitant d'une colonie (en grec, ärow A. Meillet, Dictionnaire étymologique de la langue latine. Histoire des mots (Paris 1959 $\left.{ }^{4}\right), 132$. 
étude portant sur Narbonne antique avec la célèbre inscription de l'autel au numen d'Auguste:

$\mathrm{T}$ (ito) Statilio Taur[o] / L(ucio) Cassio Longino / co(n)s(ulibus) $\mathrm{x}$ $\mathrm{K}$ (alendas) Octobr(es), / numini Augusti uotum / susceptum a plebe Narbo/nensium in perpetuom, / quod bonum faustum felixque sit $\operatorname{Imp}($ eratori) Caesari / diui $\mathrm{f}($ ilio) Augusto $\mathrm{p}$ (atri) $\mathrm{p}$ (atriae) pontifici maximo trib(unicia) potest(ate) / XxxIIII, coniugi, liberis gentique eius, senatui / populoque Romano et colonis incolisque / c(oloniae) I(uliae) $\mathrm{P}$ (aternae) $\mathrm{N}$ (arbonensis) $\mathrm{M}($ artii) qui se numini eius in perpetuum / colendo obligauerunt, plebs Narbonen/sium aram Narbone in foro posuit ad / quam quot annis viIII K(alendas) Octobr(es) qua die / eum saeculi felicitas orbi terrarum / rectorem edidit, tres equites Romani / a plebe et tres libertini hostias singu/las inmolent et colonis et incolis ad supplicandum numini eius thus et uinum / de suo ea die praestent, et VIII $\mathrm{K}$ (alendas) Octobr(es) / thus et uinum prae/stent $\mathrm{K}$ (alendis) quoque Ianuar(iis) thus et uinum / colonis et incolis praestent, viI quoq(ue) / Idus Ianuar(ias) qua die primum imperium / orbis terrarum auspicatus est thure / uino supplicent et hostias singul(as) in/molent et colonis incolisque thus ui/num ea die praestent, et pridie $\mathrm{K}$ (alendas) Iunias quod ea die T(ito) Statilio / Tauro M(anio) Aemilio Lepido co(n)s(ulibus) iudicia / plebis decurionibus coniunxit hostias / singul(as) inmolent et thus et uinum ad / supplicandum numini eius colonis et / incolis praestent exque iis tribus equitibus Roman[is tribusue] / libertinis unu[s //

[Pleb]s Narbone(n)sis a[ram] / numinis Augusti de[di]cauit [3] / [6] / [3] legibus iis q(uae) i(nfra) s(criptae) s(unt), numen Caesaris Aug(usti) $\mathrm{p}$ (atris) $\mathrm{p}$ (atriae) quando tibi / hodie hanc aram dabo dedicabo/que his legibus hisque regioni/bus dabo dedicabo quas hic / hodie palam dixero uti infimum / solum huiusque arae titulorum/que est si quis tergere ornare / reficere uolet quod beneficii / causa fiat ius fasque esto siue / quis hostia sacrum faxit qui / magmentum nec protollat id/circo tamen probe factum esto si / quis huic arae donum dare au/gereque uolet liceto eademq(ue) / lex ei dono esto quae arae est / ceterae leges huic arae titulisq(ue) / eadem sunto quae sunt arae / Dianae in Auentino hisce legi/bus hisque regionibus sicuti / dixi hanc tibi aram pro Imp(eratore) / Caesare Aug(usto) $\mathrm{p}$ (atre) $\mathrm{p}$ (atriae) pontifice maxi/mo tribunicia potestate XXXV coniuge liberis genteque eius / senatu populoque $\mathrm{R}$ (omano) colonis / incolisque col(oniae) Iul(iae) Patern(ae) Narb(onensis) Mart(ii) qui se numini eius in per/petuum colendo obligauerunt / doque dedicoque uti sies uolens / propitium. 
[Face principale] Sous le consulat de T. Statilius Taurus et de L. Cassius Longinus, dix jours avant les kalendes d'Octobre (22 septembre 11 de notre ère), vœu consacré par la plèbe de Narbonne au numen d'Auguste à perpétuité, afin qu'il soit bon, prospère et heureux pour l'empereur César Auguste, fils du Diuus, père de la patrie, grand pontife, revêtu de sa XXXIV ${ }^{\mathrm{e}}$ puissance tribunicienne, son épouse, ses enfants et sa famille, le Sénat et le peuple romain, les colons et les domiciliés de la colonie Julia Paterna Narbo Martius qui se sont engagés à rendre un culte perpétuel à son numen. La plèbe de Narbonne a élevé sur le forum à Narbonne un autel auprès duquel, chaque année, neuf jours avant les kalendes d'octobre ( 23 septembre), jour où le bonheur du siècle l'a fait naître (ce prince) pour gouverner le monde, trois chevaliers recommandés par la plèbe et trois affranchis immoleront chacun une victime et fourniront à leurs frais aux colons et aux domiciliés l'encens et le vin pour adresser des prières à son numen. Huit jours avant les kalendes d'octobre (24 septembre), ils fourniront aussi l'encens et le vin aux colons et aux domiciliés. Aux kalendes de janvier, ils fourniront aussi l'encens et le vin aux colons et aux domiciliés. Et de même, sept jours avant les ides de janvier ( 7 janvier), jour où pour la première fois il a inauguré son imperium sur le monde, ils adresseront des prières par l'encens et le vin, immoleront chacun une victime et fourniront l'encens et le vin aux colons et aux domiciliés. Et la veille des kalendes de juin (30 mai), parce qu'en ce jour, sous le consulat de T. Statilius Taurus et de M. Aemilius Lepidus, il adjoignit des juges plébéiens aux décurions, ils immoleront chacun une victime et fourniront aux colons et aux domiciliés l'encens et le vin pour adresser des prières à son numen. Et de ces trois chevaliers et de ces [trois] affranchis, un seul $[\ldots]$

[Face latérale] La plèbe de Narbonne a dédié l'autel au numen d'Auguste [...] selon les lois qui ont été écrites ci-dessus. Numen de César Auguste, père de la patrie, lorsqu'en ce jour je te donnerai et te dédierai l'autel que voici, je te le donnerai et te le dédierai suivant les lois et dans les limites que j'aurai publiquement ici, en ce jour, énoncées, de même que le sol où reposent l'autel et les inscriptions. Si quelqu'un veut nettoyer, orner, restaurer, à titre de bienfait, que cela soit permis par les lois humaines et divines; si quelqu'un fait le sacrifice d'une victime sans différer l'offrande supplémentaire, que cela soit cependant tenu pour régulier. Si quelqu'un veut faire un don à cet autel et l'enrichir, qu'il en ait la permission et que la même loi qui s'applique à l'autel s'applique aussi à ce don. Que pour cet autel et les inscriptions, les autres lois soient les mêmes que celles de l'autel de Diane sur l'Aventin. Suivant ces lois et dans ces limites, comme 
je l'ai dit, c'est à toi que, pour l'empereur César Auguste, père de la patrie, grand pontife, revêtu de la $\mathrm{Xxxv}^{\mathrm{e}}$ puissance tribunicienne, pour son épouse, ses enfants et sa famille, le Sénat et le peuple romain, les colons et les domiciliés de la Colonie Julia Paterna Narbo Martius, qui se sont engagés à rendre un culte perpétuel à son numen, je donne et dédie cet autel pour que tu sois favorable et propice. ${ }^{16}$

André Chastagnol, en analysant très précisément une documentation épigraphique qui le conduisit de la fin de la République au premier siècle de l'empire, visait à définir au mieux (et notamment au travers des données onomastiques) ce que furent coloni et incolae. Il avait en particulier bien souligné cette distinction 'paradoxale': les deux termes dérivant du même verbe - colo, colere $=$ habiter, cultiver la terre -, mais en distinguant désormais les nouveaux habitants venus du dehors, cultivateurs du sol qui leur a été attribué, exerçant seuls les droits politiques et les transmettant à leurs descendants, les coloni, des habitants libres du territoire colonial, indigènes pérégrins qui ne participaient plus à la vie politique de la cité dont ils étaient pourtant originaires. ${ }^{17}$

Dans cette première lecture, statique, de l'expression, les migrants sont les coloni et les natifs les incolae, ce que le temps passant, avec le processus de romanisation des provinciaux qui s'est mis en marche, allait totalement inverser pour parvenir à la lecture de nos sources juridiques plus tardives qui désignent désormais l'étranger résident en tant qu'incola, produit d'une mobilité au cœur d'un empire 'globalisé', pour employer une formulation qui a les faveurs de l'histoire contemporaine 'connectée' depuis une bonne dizaine d'années déjà. Cette question des domiciliés de l'intérieur et de l'extérieur, d'un glissement de sens qui s'opère au cours du premier siècle du Principat, nous permet d'aborder désormais le cœur de notre réflexion portant sur la dynamique propre du modèle juridictionnel de l'imperium Romanum.

16 La présence des coloni incolaeque dans le texte de l'Autel de Narbonne daté de 11 de n.è. $(C I L 12.4333=I L S 112)$ est tout à fait remarquable. On peut y ajouter la réflexion et les commentaires de Michel Gayraud dans son Narbonne antique des origines à la fin du III siècle (Paris 1981), 353: "quant aux incolae, il ne faut pas y voir les seuls indigènes non romanisés, mais plutôt l'ensemble des habitants domiciliés qui ne sont pas citoyens de la colonie mais qui s'y sont fixés intentionnellement."

17 Chastagnol 1996, op. cit. (n. 1), 13-25 = idem 1995, op. cit. (n. 1), 131-141, plus particulièrement pour ce qui nous concerne ici $14=132$. 


\section{Coloni et incolae, un dossier impérial. De la conception englobante et évolutive du droit romain}

La proximité sémantique de ces deux termes, étroitement associés dans un certain nombre d'inscriptions et dans quelques textes littéraires et juridiques, rend compte d'une réalité institutionnelle et sociale tout à fait prégnante. Ces deux ensembles distincts au sein des cités (qu'il s'agisse des coloniae ou des municipia, voire des ciuitates pérégrines), les coloni et les incolae, coexistent et collaborent. La reconnaissance de ces derniers est explicite, en tant qu'étrangers inscrits comme résidents (se reporter à la loi d'Osuna, Lex Vrsonensis, Lex Coloniae Genetiuae $)^{18}$ - je reviendrai pour le premier siècle de l'empire sur les cas des ueteres incolae -; ils se voient dès lors dotés d'un certain nombre de privilèges: de la participation aux distributions collectives ${ }^{19}$ à des rangs réservés

$18 C I L 2-5.1022=2.5439$ et $5439 \mathrm{a}=I L S 6087$ : chapitres XCV $($ qui colon $(i) /$ incolaeue erunt quibus); CIII (colon(os) / incolasque contributos) et CXXvi (colonos Geneti/uos incolasque hospites(que) atuentoresque).

19 Pour la désignation des ciues d'une colonie en tant que coloni, mais également comme municipes, nous pouvons relever, à propos de la colonie de Sicca Veneria (Le Kef), l'emploi très rare d'un terme pour l'autre dans une expression faisant référence aux municipes et incolae, CIL 8.1641 = ILs 6818, Sicca Veneria: P(ublio) Licinio M(arci)f(ilio) Quir(ina tribu) / Papiriano, procur(atori) / Augg(ustorum duorum) Imp(eratoris) Caes(aris) M(arci) Aureli(i) / Antonini Aug(usti) Germanici / Sarmatici maximi, p(atris) p(atriae), et / [di]ui Veri, a rationibus cui / splendissimus ordo Siccen/sium ob merita eius et cu[ram(?) 3] / [3] M et [3] / [6] / M[3]N[3] / [6] / [6] / [3]LEC[3] / [3]T[3] / [3]II[ // Municipibus meis Cirthensibus / Siccensibus carissimis mihi dare / uolo HS MCCC(milia). Vestrae fidei committo, / municipes carissimi, ut ex usuris / eius summae quincuncibus quodan/nis alantur pueri CCC et puellae CC, pueris / ab annis tribus ad annos XV et accipiant / singuli pueri |(denarios) II s(emissem) menstruos, puellae / ab annis tribus ad annos XIII |(denarios) II. Legi / autem debebunt municipes item in/colae, dumtaxat incolae qui intra / continentia coloniae nostrae ae/dificia morabuntur; quos, si uo/bis uidebitur, optimum erit per / IIuiros cuiusque anni legi; cura/ri autem oportet, ut in locum ad/ulti uel demortui cuiusque sta/ tim substituatur ut semper ple/nus numerus alatur. "A Publius Licinius Papirianus, fils de Marcus, de la tribu Quirina, procurateur a rationibus des deux Augustes, l'empereur César Marcus Aurelius Antoninus Auguste Germanicus Très grand Sarmatique, père de la patrie, et le divin Verus, à qui le très splendide ordre des habitants de Sicca pour ses mérite et soin [...] (Sur le côté gauche) A mes très chers concitoyens (municipes) de Cirta Sicca, je veux donner 1,300,000 sesterces. Je m'en remets à votre bonne foi, très chers municipes, pour qu'avec les intérêts aux $5 / 12^{\mathrm{e}}$ de la somme chaque année on nourrisse 300 garçons et 200 filles; que les garçons, de 3 à 15 ans, reçoivent chacun 2,5 deniers par mois; les filles, de 3 à 13 ans, chacune 2 deniers par mois. Et l'on devra choisir des citoyens ainsi que des résidents, pourvu que les résidents demeurent dans l'agglomération de notre colonie. Ceux-ci, si vous l'acceptez, il sera mieux qu'ils soient choisis par les duumvirs 
au théâtre, jusqu'à des droits judiciaires, voire au droit de vote, enfin à l'admission dans la curie (l'ordre des décurions), comme l'attestent les inscriptions de Die et Axati. On peut s'appuyer, pour quelques exemples significatifs, sur les relevés de François Bérard, à propos notamment de Sex. Vencius Iuventianus, un notable admis dans la curie en tant que résident étranger:

Dea Augusta Vocontiorum: Sex(to) Vencio / Iuuentiano, / flamini diui Aug(usti) / item flamini et cura/tori muneris gladi/atori(i) Villiani, adlec/ to in curiam Lugudu/nensium (sic!) nomine / incolatus a splen/didissimo ordine / eorum, / ordo Vocontior(um) / ex consensu et pos/tulatione populi / ob praecipuam / eius in edendis / spectaculis li/beralitatem.

A Sextus Vencius Iuventianus, flamine du divin Auguste, et aussi flamine et curateur du combat de gladiateurs Villianus, agrégé dans la curie des Lyonnais en tant que résident par leur très splendide ordre, l'ordre des Voconces, avec l'accord et sur demande du peuple, en raison de sa particulière libéralité dans l'organisation des spectacles. ${ }^{20}$

avec le parallèle du municipe flavien d'Axati, et l'exemple de L. Lucretius Severus, ex incolatu decurioni:

L(ucio) Lucretio Seuero / Patriciensi / et in municipio / Flauio Axatitano / ex incolatu decurioni / statuam quam testamento / s(uo) poni sibi iussit / datis sportulis / decurion[ibus m(unicipum)] / m(unicipii) F(laui) Axatitani [3] / [3]simus XII[.

A Lucius Lucretius Severus, Patriciensis et décurion dans le municipe flavien d'Axati parmi les résidents, la statue que, dans son testament, il a ordonné qu'on lui élève; des sportules ont été distribuées aux décurions des municipes du municipe flavien d'Axati [---]. ${ }^{21}$

de chaque année, et il faudra aussi se préoccuper de remplacer sans attendre les adultes ou les défunts, pour qu'on nourrisse toujours un groupe complet." Pour deux analyses de cette fondation alimentaire qui bénéficie aux deux composantes de la cité: $\mathrm{M}$. Christol et A. Magioncalda, 'La fondazione di P. Licinio Papiriano da Sicca Veneria', L'Africa Romana 8 (1991), 321-330, et N. Kallala, 'À propos de la fondation de P. Licinius Papirianus de Sicca Veneria (CIL VIII, 1641): Quelques mises au point', Africa 20 (2004), 109-120. 
et le dossier des droits politiques octroyés aux incolae, notamment enrichi par le chapitre 53 de la Lex Flauia Malacitana:

[53] r(ubrica) in qua curia incolae suffragia / ferant / quicumque in eo municipio comitia Iluiris / item aedilibus item quaestoribus rogan/dis habebit ex curiis sorte ducito unam / in qua incolae qui ciues $\mathrm{R}$ (omani) Latiniue ciues / erunt suffragi $\{0\}<u m>$ ferant eisque in ea cu/ria suffragi latio esto / (...)

Dans quelle curie votent les incolae. Celui qui tiendra les comices dans ce municipe pour élire les duumvirs, ainsi que les édiles, de même que les questeurs, devra tirer au sort l'une des curies dans laquelle voteront les résidents, qu'ils soient citoyens romains ou citoyens latins, et ils auront le droit de vote dans cette curie. ${ }^{22}$

De fait, lévolution de la signification du terme incola rend bien compte des mutations de la société romaine impériale et de sa capacité à l'intégration sociale, puis juridique, de populations 'indigènes' confrontées aux résultats de la conquête de territoires, de la déduction de colonies avec installation - parfois multiple - de vétérans ou de plébéiens civils, ${ }^{23}$ à l'installation dans des communautés de type romain d'étrangers domiciliés et reconnus comme tels, au-delà du laps de temps jugé nécessaire (voir n. 14 supra). Ces migrants de l'extérieur que le droit définit très précisément au deuxième siècle avec Pomponius et Gaius, ${ }^{24}$ en tant que domiciliés, devant obéissance aux magistrats, en particulier

22 CIL 2. $1964=I L S 6089$. Mobilité géographique et mobilité sociale, à propos des incolae admis à la curie: F. Bérard, 'L’organisation municipale de la colonie de Lyon', in M. DondinPayre et M.-Th. Raepsaet-Charlier, eds., Cités, municipes, colonies. Les processus de municipalisation en Gaule et en Germanie sous le Haut Empire romain. Coll. Histoire ancienne et médiévale 53 (Paris 1999, 2 $2^{\mathrm{e}}$ tirage 2009), 97-126, ici 108-109. De manière générale, on renverra à la synthèse des données concernant le fonctionnement des institutions des cités que l'on doit à M. Dondin-Payre, 'Magistratures et administration municipale dans les Trois Gaules', in M. Dondin-Payre et M.-Th. Raepsaet-Charlier, eds., Cités, municipes, colonies. Les processus de municipalisation en Gaule et en Germanie sous le Haut Empire romain. Coll. Histoire ancienne et médiévale 53 (Paris 1999, $2^{\mathrm{e}}$ tirage 2009), 127-230.

23 On peut citer l'exemple de Narbonne, avec une installation de civils en 118, puis de vétérans de la Xe légion en 45. Voir Gayraud 1981, op. cit. (n. 16).

24 Les dispositions du droit romain (au deuxième siècle), à propos des domiciliés d'origine étrangère sont les suivantes: Incola est, qui aliqua regione domicilium suum contulit; quem Graeci paroikon appellant "Est résident, celui qui place son domicile dans telle région; c'est ce que les Grecs appellent 'paroikon' (voisin, habitant auprès de)", Dig. 50. 16 
le respect de leurs charges politiques et fiscales, sont donc une composante des ciuitates, dans le cadre d'une évolution remarquable des statuts des hommes et des communautés tout au long de ces deux premiers siècles de l'empire. Ainsi, la figure impériale est souvent présente quand il s'agit de mesurer la composition mixte de ces communautés: qu'il s'agisse de l'autel de Narbonne, en 11 de notre ère, qui permet d'attester l'association étroite des domiciliés (étrangers ou indigènes) au culte organisé en l'honneur du numen d'Auguste (se reporter supra au texte cité) avec toutes les implications d'un calendrier local aux fortes connotations romaines - ce qui participe de ce mouvement de construction d'une communis patria 'impériale', au sens où l'empereur en est le garant et le promoteur -, ou bien du témoignage d'Aoste, dans une dédicace à Auguste patron de la colonie qui mentionne les Salassi incolae présents depuis l'origine (initio). ${ }^{25}$ La fondation de Terentius Varron en 25 (Augusta Praetoria), sur l'emplacement de son camp militaire, comprend donc l'installation de 3,00o vétérans, selon Strabon et Dion Cassius, ainsi que des Salasses qui devaient faire partie de ceux qui avaient fait reddition avant la fin du conflit, hommes libres inscrits aux côtés des coloni. ${ }^{26}$ La dédicace date des tout débuts de la colonie, en 23-20 avant notre ère. Cet exemple permet d'évoquer à propos de ces 'étrangers de l'intérieur' le sort des vaincus et les déplacements de population que cela a pu générer $(36,000$ personnes, dont 8,00o combattants, furent vendus comme esclaves, toujours selon nos sources littéraires).

Le dossier d'Avenches ${ }^{27}$ me permet de terminer cette évocation de la mention des coloniet incolae dans les cités de l'Occident romain au premier siècle de l'empire, le glissement de sens sur lequel nous reviendrons en dernière partie,

(De uerborum significatione), 239. 2 (Pomponius libro singulari enchiridii); Incola et his magistratibus parere debet, apud quos incola est, et illis, apud quos ciuis est, "Le résident doit à la fois obéir aux magistrats auprès desquels il est résident et à ceux auprès desquels il est citoyen", Dig. 50. 1 (Ad municipalem et de incolis), 29 (Gaius 1 ad edictum prouinciale).

25 Pour le cas de la colonie d'Aoste, ILs 6753 = IIt. 11.1, 6; A.M. Cavallaro et G. Walser, Iscrizioni di Augusta Praetoria (Aoste 1988), no. 1, Augusta Praetoria: Imp (eratori) Caesa[ri] / diuif(ilio) August(o) / co(n)s(uli) XI, imp(eratori) VI[II], / tribunic(ia) pot(estate), / Salassi incol(ae), / qui initio se / in col(onia) con[t(ulerunt)], / patron(o). "A l'empereur César Auguste, fils du divin, consul onze fois, salué imperator huit fois, revêtu de la puissance tribunicienne, les résidents Salasses, qui sont placés depuis son origine dans la colonie, à leur patron."

26 Strab. 4. 6.7 et C.D. $53 \cdot 25 \cdot 3-5$.

27 À propos de la colonie d'Avenches, se reporter à l'article de R. Frei-Stolba, avec A. Bielman et H. Lieb, 'Recherches sur les institutions de Nyon, Augst et Avenches', in M. DondinPayre et M.-Th. Raepsaet-Charlier, eds., Cités, municipes, colonies. Les processus de municipalisation en Gaule et en Germanie sous le Haut Empire romain. Coll. Histoire ancienne et médiévale 53 (Paris 1999, 2 e tirage 2009), 29-95, part. 'La ciuitas Heluetiorum', 67-91. 
des étrangers de l'intérieur aux étrangers domiciliés, et l'épineuse question du statut des communautés que je ne souhaite pas aborder de front dans le cadre de cette brève réflexion - colonies romaines, colonies latines et municipes de droit latin. Toutefois, deux expressions, qui ont entraîné de nombreux débats dans la communauté scientifique, vont me permettre de poser les jalons de ma toute dernière partie conclusive. Il s'agit des trois curatores col. connus, avec notamment l'exemple de T. Tertius Severus, et des patroni publici. ${ }^{28}$ Dans le premier cas, il s'agit de trois dédicaces à la déesse tutélaire de la colonie, Dea Auentia, qui mentionnent quatre personnages dont les profils s'apparentent à des incolae - un Trévire est nommément attesté. ${ }^{29} \mathrm{Si}$ l'on a proposé de développer en curator colonorum (Patrick Le Roux en dernier lieu), il s'agirait alors d'une association de colons déduits et installés à Avenches, considérée comme une colonie romaine, ce qui pose le problème du profil des curateurs et de la présence des incolae. On rejettera de même la solution d'une simple haplologie, curator (incolarum) col(oniae), et l'on préférera la cura confiée à un riche individu, incola qui se voit ainsi reconnu, à défaut de pouvoir faire une carrière de magistrat. ${ }^{30}$ Ce qui serait également le sens de ces patroni publici, pris

28 Concernant les coloni Auenticenses, les incolae Auenticenses, les curator col(-) et les patroni publici, Frei-Stolba 1999 op. cit. (n. 27), 85-88, 'Les curatores col(-)'

CIL 13. $5073=$ ISchweiz $207=$ RIS 75, Avenches: Deae Auentiae / et Gen(io) incolar(um) / T(itus) Ianuarius / Florinus / et P(ublius) Domitius / Didymus, / curatores col(oniae), ex stipe annua / adiectis de suo / HS n (ummum) I(mille)D. "A la déesse Aventia et au Génie des incolae, Titus Ianuarius Florinus et Publius Domitius Didymus, curateurs de la colonie, avec les revenus annuels, ajoutant à leurs frais la somme de 1,500 sesterces." CIL 13.5071 = ISchweiz 209, Avenches: Deae Auent(iae) / C(aius) Iul(ius) Primus / Treuir, / cur(ator) col(oniae), item / cur(ator) IIIIIIuir(um), / de suo posuit / [6] / [3]RII[3] / l(ocus) d(atus) d(ecreto) [d(ecurionum $)]$. "A la déesse Aventia, Caius Iulius Primus, Trévire, curateur de la colonie, de même curateur des sévirs, a posé à ses frais [...], emplacement donné par décret des décurions." CIL 13. $5072=$ ISchweiz 208 = RIS 74, Avenches: Deae Auent (iae) $/ T($ itus $)$ Tertius /Seuerus, / cur(ator) colon(iae)/idemque all(ector), / cui incolae / Auenticens(es) / prim(o) omnium / ob eius erga / se merita / tabulam arg(enteam) / $p[u b] l($ ice?) / posuer(unt) / donum d(e) s(ua) p(ecunia) / ex HS V(milibus)CC, l(ocus) d(atus) d(ecreto) d(ecurionum). "A la déesse Aventia, Titus Tertius Severus, curateur de la colonie, et de même membre du collège, auquel, le premier de tous, les résidents d'Avenches, pour ses mérites envers eux, ont posé cette table d'argent publiquement, a fait don sur ses propres fonds de 5,200 sesterces, emplacement donné par décret des décurions."

30 Résumé des différentes positions par Frei-Stolba 1999, op. cit. (n. 27), 85-87, avec notamment successivement, pour les trois hypothèses proposées: P. Le Roux, 'La question des colonies latines sous l'Empire', Ktéma 17 (1992), 183-200; P. Frei, 'Zur Gründung und zur Rechtsstellung der römischen Kolonie Aventicum', Bulletin de l'Association Pro Aventico 20 (1969), 5-22 et finalement J. Krier, Die Treverer ausserhalb ihrer Civitas (Trèves 1981). 
sur place, d'origine locale, aux côtés des grands personnages, marquant ainsi des liens étroits entre coloni et incolae Auenticenses. ${ }^{31}$ Dans cette approche, les mécanismes propres au droit latin ont par ailleurs, en un temps relativement court, concouru à une unification des statuts - par le biais d'une diffusion régulière de la ciuitas Romana, par le ius Latii puis le Latium maius -, et une fusion des communautés - le statut des incolae changeant radicalement de signification au fur et à mesure de la diffusion de la citoyenneté romaine --, sur lesquelles je souhaiterai achever mon propos.

Fiction juridique, dynamique intégrative et conception de l'Imperium Romanum, de l'hétérogénéité à l'homogénéité: remarques conclusives en marge de deux livres récents de Clifford Ando

Un premier constat s'impose, qui est riche de sens pour l'enquête commune engagée sur mobilité et migration: les mécanismes de l'expansion romaine se fondent sur des jeux de miroir, d'identités en regard. Le migrant (le colon romain) s'enracine dans un territoire et transforme les populations locales en résidents, partageant une communauté, sinon en droit, et se fondant à terme dans une collectivité plus large d'étrangers résidents, les nouveaux migrants dont les statuts personnels peuvent varier, du pérégrin au citoyen romain, ceux-là même qui devront justifier d'une durée minimale d'installation dans la cité.

La fictio ciuitatis qu'étudie C. Ando, dans la littérature jurisprudentielle (avec Gaius notamment), s'accompagne de rapports structurants qui permettent de penser la communis patria en autant de couples antithétiques, qui sont voués à une certaine fusion, au terme de trois à quatre siècles d'histoire impériale: ciuis/peregrinus (avec les effets de l'édit de Caracalla, ou Constitutio Antoniniana); colonus/ incola (dans une dynamique des échanges), réductible à l'identification ciuitas/ Imperium Romanum, ce qui pose en définitive le

$31 \quad$ CIL 13.5091 = ISchweiz $211=$ RIS 83, Avenches: $]$ IEIVI [3] / quaestura in $p[$ rou $($ incia $)] /$ Asia functu[s est], / incolae Auent[ic]ens(es) / ob egreg(ia) eius erga se mer(ita) / patron(o) p(ublice), cui singuli / adq(ue) uniuers(i) obligatos / se esse praeferunt adq(ue) / etiam parum sibi ui/dentur praedicare / cura T(iti) Nigr(ini) Modesti / IIIIIIuiri Augustalis. "[---] il a accompli la questure dans la province d'Asie, les résidents d'Avenches, en raison de ses remarquables mérites envers eux, à leur patron, publiquement, auquel ils se sont présentés comme étant ses obligés, à titre indivituel comme à titre collectif, tout comme ils semblent le célébrer, sous la conduite de Titus Nigrinus Modestus, sévir augustal." 
problème du rapport entre Rome et les cités provinciales, et la question d'une conception globale, universelle, d'un paradoxe de l'exclusion qui aboutit à l'inclusion (saluo iure patroni, saluo iure gentis). Les productions normatives impériales concourent à cette mutatio stationis, tant à l'échelle individuelle qu'au stade collectif. L'empire, cité universelle, se construit à partir d'une pluralité de statuts personnels et collectifs et de processus de fusion.

En définitive, j'adhère volontiers à l'idée d'une production de l'homogénéité à partir de l'hétérogénéité, mais interprète différemment les réflexions d'Hadrien, rapportées par Aulu-Gelle et analysées par C. Ando:

Sed coloniarum alia necessitudo est; non enim ueniunt extrinsecus in ciuitatem nec suis radicibus nituntur, sed ex ciuitate quasi propagatae sunt et iura institutaque omnia populi Romani, non sui arbitrii, habent. Quae tamen condicio, cum sit magis obnoxia et minus libera, potior tamen et praestabilior existimatur propter amplitudinem maiestatemque populi Romani, cuius istae coloniae quasi effigies paruae simulacraque esse quaedam uidentur, et simul quia obscura oblitterataque sunt municipiorum iura, quibus uti iam per ignotitiam noli queunt.

Mais ce qui lie les colonies est différent: car elles ne viennent pas de l'extérieur pour entrer dans le corps citoyen et elles ne sont pas nourries par leurs propres racines, mais elles sont propagées à partir du corps citoyen lui-même et ont toutes les lois et les institutions du peuple romain, sans les avoir elles-mêmes choisies. Ce statut, cependant, bien que plus pesant et moins libre, est pourtant considéré comme préférable et plus digne, si l'on tient compte de la magnificence et de la grandeur du peuple romain, dont ces colonies semblent être l'image et des sortes de copies; et, en même temps, parce que les lois des municipia sont obscures et oubliées, par ignorance, celles-ci ne sont pas en état d'en faire usage. ${ }^{32}$

Que dire donc de cette aspiration au statut colonial face au statut municipal, jugé pourtant plus libre, dans ce qui apparaît comme une sorte de cursus honorum menant de la ciuitas à la colonie dotée du ius Italicum, autre avatar de la fiction juridique et spatiale de la communis patria?33

32 Aul.Gel. NA 16.13. 8-9. De la colonie, du municipe, de la conception des statuts personnels, des communautés et de l'Imperium Romanum, en se reportant à Ando 2013, op. cit. (n. 9), 230.

33 Au sens que le Digeste avec Modestin lui assigne: Roma communis nostra patria est (Herennius Modestinus, Manualia, 1 = Dig. 50.1.33; également Dig. 27.1.6.7). On se reportera 
Le rapport entre coloni et incolae et celui entre colonies et municipes m’apparaît du même ordre, de la différence à l'identité, du pareil au même, dans un mouvement paradoxal de la mobilité des statuts, de la migration flux vital - au sein d'une cité universelle, mouvement nécessaire qui, une fois brisé, renvoie l'utopie à l'émiettement tardo-antique. Dans cette perspective, l'histoire continue et le quatrième siècle, sur lequel nous avons ouvert cette réflexion, permettrait d'analyser le devenir des fictions juridiques et les effets des migrations des peuples, réinventant une nouvelle approche de la cité impériale, au-delà des ruptures effectives ou fluctuantes des partes occidentalis et orientalis. ${ }^{34}$

Rome-Paris, Juin 2015-Février 2016

aux développements de Thomas 1996 op. cit. (n. 13). Pour une remise en perspective impériale d'une évolution sur la longe durée, $\mathrm{S}$. Benoist, 'Le prince, magister legum: réflexions sur la figure du législateur dans la Rome impériale', in P. Sineux, ed., Le législateur et la loi dans l'Antiquité. Hommage à Françoise Ruzé (Caen 2005), 225-240, complété par idem, 'Penser la limite: de la cité au territoire impérial', in O. Hekster et T. Kaizer, eds., Frontiers in the Roman World. Proceedings of the Ninth Workshop of the International Network Impact of Empire (Durham, 16-19 April 2009) (Leyde et Boston 2011), 31-47.

34 On pourrait tirer ainsi profit, pour la présente réflexion, d'une lecture affinée de l'Expositio totius mundi et gentium et des mouvements successifs de codification impériale. On peut prendre en outre un seul exemple avec cette étude récente de l'évolution tardo-antique de la Gaule dans ce cadre: A.E. Jones, Social Mobility in Late Antique Gaul: Strategies and Opportunities for the Non-elite (Cambridge et New York 2009). 